\title{
Statistical investigations of an ENIG Nickel film morphology by Atomic Force Microscopy
}

\author{
Rosine Coq Germanicus ${ }^{1,3}$, Philippe Leclère ${ }^{2}$, Eric $\operatorname{Hug}^{3}$, Florent Lallemand ${ }^{4}$, \\ and Philippe Descamps ${ }^{1}$ \\ ${ }^{1}$ Normandie Université ENSICAEN / CRISMAT / UMR 6508, 6 boulevard Maréchal Juin, \\ 14050 Caen Cedex 04, France \\ ${ }^{2}$ Service de Chimie des Matériaux Nouveaux, CIRMAP, Université de Mons, Belgium \\ ${ }^{3}$ CRISMAT, Normandie Université, UMR CNRS 6508, 14000 Caen, France \\ ${ }^{4}$ IPDiA, 2 rue de la girafe, 14000 Caen, France
}

\begin{abstract}
The morphology of a Nickel layer grown by an Electroless Nickel Immersion Gold (ENIG) technique used for microelectronics interconnections is determined by Atomic Force Microscopy (AFM) investigations. The root mean square (rms) roughness, determined over a scanned area is a function of the AFM scanned area size. In this work, we propose to consider the dynamic scale theory and the power spectrum density (PSD) analysis in order to perform a comprehensive determination of the surface properties of the ENIG nickel layer. Results highlight the existence of a first regime with a roughness exponent of 0.95 and a fractal dimension (DF) of the nickel film about 2.05. This case study is presented in order to propose further investigations. In fact, same experimental procedure should be performed in a magnetic shielded zone where a very low noise level is available such as the Low-Noise Underground Laboratory (LSBB) of Rustrel (France).
\end{abstract}

\section{Introduction}

In order to explore optical, mechanical, electrical and chemical properties of surfaces, quantitative investigations of surface morphology are important requirements to perform comprehensive studies. At the nanoscopic scale, Atomic Force Microscopy (AFM) appears to be a useful research tool in a wide range of different scientific fields such as materials, physics, chemistry, geology, and biology [1-4]. The AFM probe consists in a cantilever equipped with a sharp tip with a diameter of $5-30 \mathrm{~nm}$. Based on the interactions between this tip and the probed surface, the deflection of the cantilever is measured and controlled via an optical system. While the tip scans the surface, a feedback loop is used to determine the topography when the system is maintaining a constant deflection value. In the last 30 years, AFM instruments have been developed to easily obtain 3D-mapping surface topographies and nanostructures with stable and high signal-to-noise ratio regarding the $\mathrm{X}, \mathrm{Y}$ and $\mathrm{Z}$ resolution. The vertical resolution $(Z)$, called sensitivity, is determined by the thermal and acoustic noise of the cantilever. For a free rectangular cantilever with a spring constant $\mathrm{k}$ of $1 \mathrm{~N} / \mathrm{m}$, when measurements are performed at room temperature in standard laboratory environment, the thermal noise is near $0.074 \mathrm{~nm}$ [5]. The lateral resolution $(\mathrm{X}, \mathrm{Y})$ depends on the tip apex geometry and on the tip convolution with the surface. 


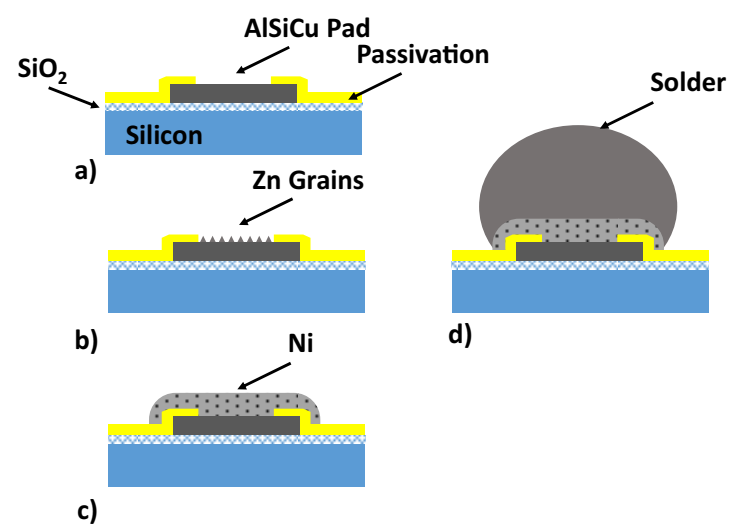

Figure 1. Schematic of the ENIG process for interconnections with Solder a) AlSiCu Pad, b) Zincation pre-plating, c) ENIG, d) Solder ball.

From topographic images, for a first order statistics, the morphology of the sample is often characterized by the Root Mean Square (RMS) roughness. This statistic parameter is determined over the fixed scanned area. For more complex film morphologies, this unique value cannot be sufficient. For deeper studies, dynamic scale analysis and Power Spectral Density (PSD) were developed [6] to describe more accurately the surface properties. The analysis is based on the self-similarity or self-affinity properties [7]. In this study, we propose to use the dynamic scale analysis of a nickel layer surface grown in a microelectronic cleanroom as an UBM (Under Bump Metallization) for interconnections.

\section{Experimental details}

In order to realize interconnections for highly integrated circuits, the Electroless Nickel Immersion Gold (ENIG) technique is widely used in microelectronic before components assembly [8]. The goal of this process consists in obtaining a Nickel film on interconnections pads, with a high uniformity and excellent conductivity. The main steps of ENIG process is depicted on Fig. 1. The surface morphology of the nickel film is a critical parameter for Back End of Line (BeOL) [9]. In the studied sample, the metal finishing is an aluminium alloy $(\mathrm{AlSiCu})$ deposited on bond pads (Fig. 1a). The diameter of the pad is fixed at $50 \mu \mathrm{m}$. In a second step, a pre-plating process called "zincation" activates the metallic surface (Fig. 1b). Then two metallic layers: a thick nickel coated with a thin film of gold are grown on the pads (Fig. 1c). This UBM represents the interfacial layer between the metal finishing and the solder balls (Fig. 1d) used for connecting the integrated circuit (IC) with the package or with an over IC. The electroless time is controlled in order to create a nominal nickel thickness of typically $5 \mu \mathrm{m}$ on the pad.

In this study, the ENIG process was stopped after the nickel growth. As a consequence, the thin gold film that is typically coated on the nickel is not investigated here. The microstructure of the deposit is examined by Scanning Electron Microscopy (SEM) and AFM. The AFM images are recorded in Tapping mode using a D3100 AFM (Bruker Nano Inc. Santa Babara, CA, USA) under standard atmospheric conditions. Commercial tips with a radius of about $8 \mathrm{~nm}$ were used. AFM images of $512 \times 512$ pixels were obtained. The scan rate was fixed at $0.5 \mathrm{~Hz}$. Then, from acquisitions, film morphology and roughness were determined by using the Nanoscope software (Nanoscope 1.5). 


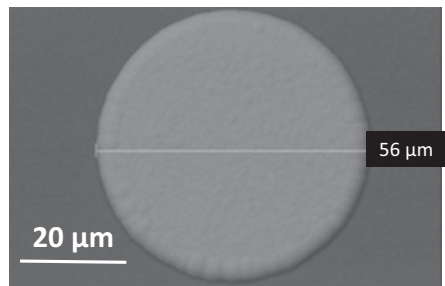

a)

Figure 2. SEM images of the Nickel surface on pads.

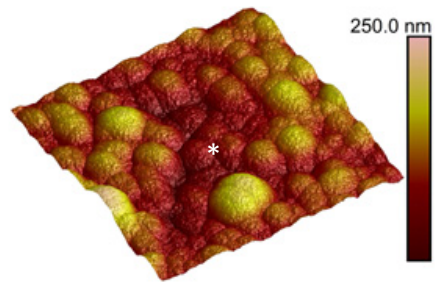

a) $10 \mu \mathrm{m} \times 10 \mu \mathrm{m}$

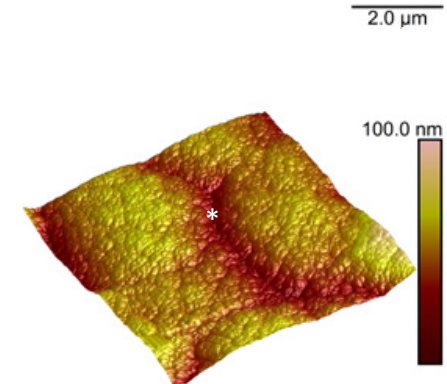

c) $2 \mu m \times 2 \mu m$ b) $5 \mu m \times 5 \mu m$

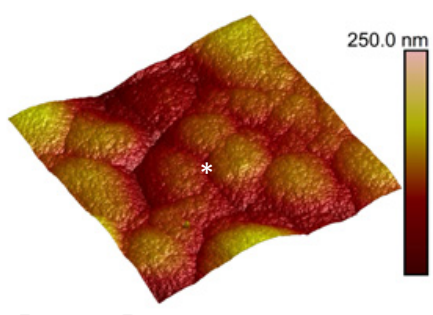

b)
$5 \mu \mathrm{m}$ 


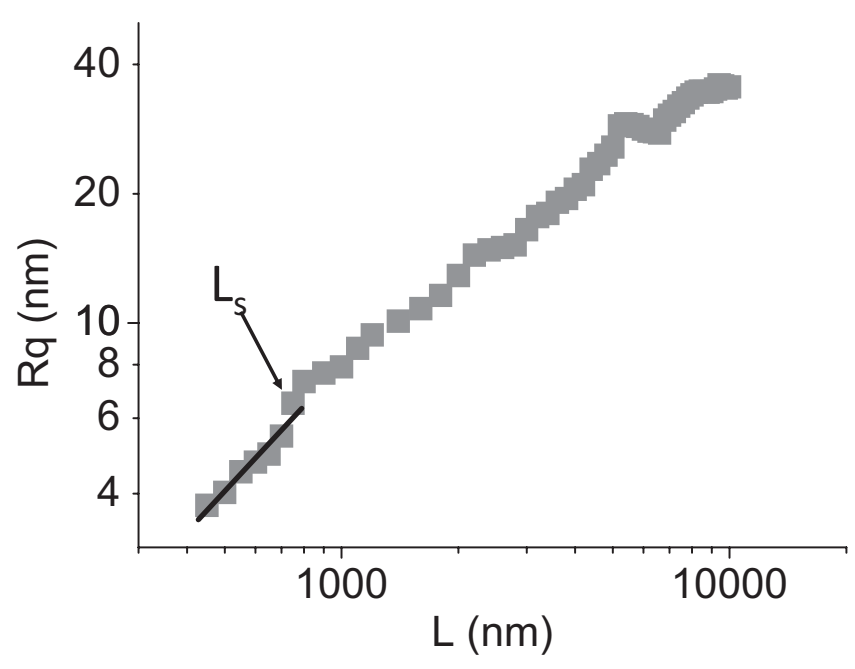

Figure 4. Plot of the roughness as a function of the scan size L.

Typically, at a first order statistic, the film microstructure is characterized by the Root Mean Square (RMS) roughness, also called Rq, which represents the standard deviation of surface heights. The average roughness $\mathrm{Ra}$ which indicates the height deviation is also a parameter considered for roughness analysis. The evolution of both $\mathrm{Ra}$ and $\mathrm{Rq}$ parameters determined inside the fixed 3D scanned area as a function of the surface size and also depending of the image pixel surface. In order to determine the film morphology, the dynamic scale theory [10] is used to define the RMS roughness law as a function as the image size. The plot of the roughness against the scanning size is shown in Fig. 4, this curve is derived out from the AFM topographic image $(10 \mu \mathrm{m} \times 10 \mu \mathrm{m})$ presented in Fig. 3. In this case the analysis is performed with a constant surface per pixel. The graphic highlights the existence of a first scale regime. This first regime represents the film properties within an individual grain. The correlation length $\mathrm{L}_{\mathrm{s}}$ is estimated to be about $750 \mathrm{~nm}$. This value is in agreement with the average diameter of the grain. The roughness exponent define by $R q(L) \sim L^{\alpha}$ is equal to 0.95 . The fractal dimension of the film, is then determined at 2.05 (with $\mathrm{DF}=$ $3-\alpha$ ). From [11], the analysed surface can be considered that a relative smooth surface.

We also applied the Power Spectral Density (PSD) analysis. PSD relates the Fourier transform of the AFM image in spatial domain [12]. PSD functions are calculated from AFM measurements obtained for the four scan sizes from $1 \mu \mathrm{m} \times 1 \mu \mathrm{m}$ to $10 \mu \mathrm{m} \times 10 \mu \mathrm{m}$. This analysis provides also information on the lateral roughness distribution. The PSD results versus spatial frequency are plotted on log-log scale in Fig. 5. For high spatial frequencies, the slope of the log-log plot $\mathrm{H}$ provides the fractal dimension, deduced from $\mathrm{DF}=4-\mathrm{H} / 2$. The calculation results $\mathrm{DF}=2.06$ in agreement with the dynamic scale analysis.

Calculated in 3-D, this DF value seems indicated that the deposition mechanism is a diffusion-limited growth [13]. The fractal dimension analysis is performed to evaluate quantitatively a single surface parameter $\mathrm{q}$ for the studied surface. Our motivation is to propose the same experimental analysis in the Low-Noise Underground Laboratory (LSBB) in order to determine the impact of the noise in topography AFM analysis. We propose to perform AFM measurements for a similar surface in the LSBB in order to probe surface properties in static or dynamic modes. The goal should be to compare ground and underground analysis and quantify the gain in terms of noises in the LSBB environment. 


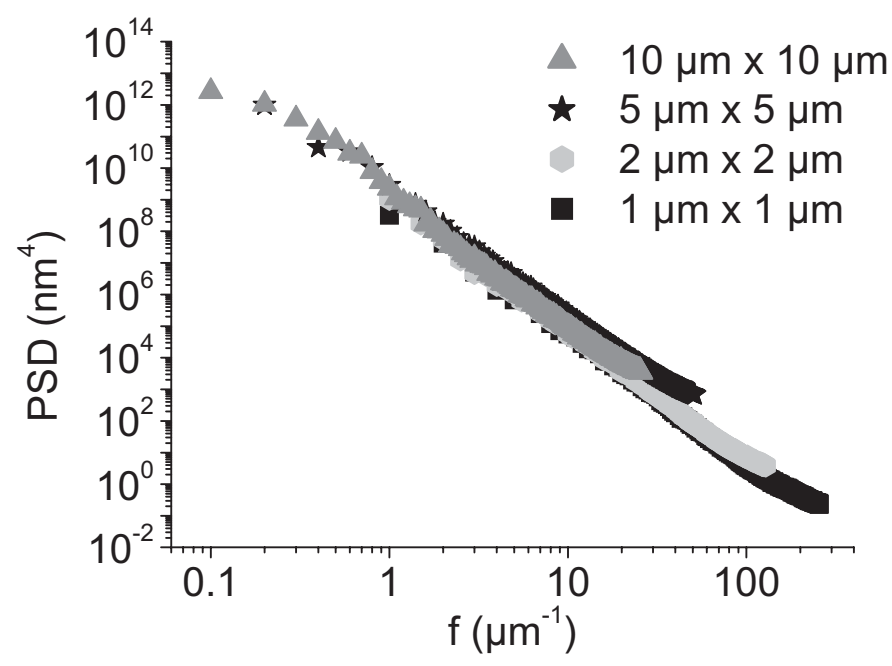

Figure 5. PSD results for the four scan sizes.

\section{Conclusion}

In this study, we have explored a comprehensive determination of the surface morphology of an ENIG nickel layer grown for microelectronics BEoL process. From AFM acquisitions, the dynamic scale theory and the PSD analysis were applied to determine the roughness exponent and the fractal dimension. The results indicate a roughness exponent of 0.95 and a fractal dimension of the nickel film of about 2.05 with a good correlation between the two techniques. Nevertheless, since experiments presented in this paper are performed in a standard conditions, potential electromagnetic noise might have impacted the recorded data. Thus in order to increase the accuracy on this methodology for fractal surface analysis, further investigations should be performed in a magnetic shielded zone where a very low noise level is available such as the LSBB of Rustrel (France). These very specific environment conditions, where noisy sources are reduced, should be able to provide a far better sensitivity.

\section{References}

[1] P. Eyben, W. Vandervorst, D. Alvarez, M. Xu, and M. Fouchier, Scanning Probe Microscopy, Springer New York, 31 (2007).

[2] R. Coq Germanicus, E. Picard, B. Domenges, K. Danilo and R. Rogel, Applied Surf. Sci. 253, 6006 (2007).

[3] L. Leyssenne, S. Wane, S. Massenot, D. Bajon, R. Coq-Germanicus, P. Descamps and G. Audoit, ICEAA 1032 (2013).

[4] Ph. Leclère, P. Viville, M. Jeusette, J.P. Aimé and R. Lazzaroni, In Scanning Probe Microscopies: beyond imaging, 175 (Wiley-VCH, 2006).

[5] Y. Gan, Surface Sci. Rep. 64, 99 (2009).

[6] L. Vázquez, R.C. Salvarezza, P. Herrasti, P. Ocón, J.M. Vara and A.J. Arvia, Applied Surf. Sci. 70 (1993).

[7] R.R.L. De Oliveira, D.A.C. Albuquerque, F.L. Leite, F.M. Yamaji, and T.G.S. Cruz, Measurement of the nanoscale roughness by atomic force microscopy: basic principles and applications INTECH (2012). 
[8] B.K. Kim, S.J. Lee, J.Y. Kim, K.Y. Ji, Y.J. Yoon, M.Y. Kim, S.H. Park, and J.S. Yoo, Journal of Electr. Mat., 37(4), 527 (2008).

[9] J. Wojewoda-Budka, Z. Huber, L. Litynska-Dobrzynska, N. Sobczak and P. Zieba, Mat. Chem. and Phys. 139, 276 (2013).

[10] N.C. De Souza, J.R Silva, M.A. Pereira-da-Silva, M. Raposo, R.M. Faria, J.A. Giacometti, Journal of Nanosci. and Nanotech, 4(5), 548 (2004).

[11] A.P. Pentland, IEEE transactions on pattern analysis and machine intelligence, 6, 661 (1984).

[12] F. Biscarini, P. Samori, A. Lauria, P. Ostoja, R. Zamboni, C. Taliani, P. Viville, R. Lazzaroni and J.L. Bredas, Thin Solid Films, 284, 439 (1996).

[13] T. Vicsek, Fractal growth phenomena 2, Singapore: World scientific (1992). 